\title{
Lane Detection Method of Statistical Hough Transform Based on Gradient Constraint
}

\author{
Peng Yan-zhou, Gao Hong-feng* \\ College of Information Engineering, Henan University of Science and Technology, Luoyang, China
}

Email address:

pengyanzhou@sina.com (Peng Yan-zhou), gaohongfenghappy@126.com (Gao Hong-feng)

To cite this article:

Peng Yan-zhou, Gao Hong-feng. Lane Detection Method of Statistical Hough Transform Based on Gradient Constraint. International Journal of Intelligent Information Systems. Vol. 4, No. 2, 2015, pp. 40-45. doi: 10.11648/j.ijiis.20150402.12

\begin{abstract}
A lane detection method of statistical Hough transform based on gradient constraint is proposed to solve the problem of computational cost and grid quantization precision of classical Hough transform. Statistical Hough transform uses the Gaussian kernel function to model each pixel in the image. The size of initial data set is limited by using the method of gradient constraint. Eventually lane parameters' continuous probability density function is given. The results of the experimentation show that under highway circumstance the provided method can rapidly and robustly detect the lane.
\end{abstract}

Keywords: Statistical Hough Transform, Gaussian Kernel Function, Gradient Constraint

\section{Introduction}

With the increase in the number of vehicles, the number of car accident on highway has increased annually. Many accidents are caused by a lack of awareness about driving conditions due to driver carelessness or visual interference. Lane detection and tracking technology based on visual are the key technology on the applications of driver assistance systems for automobiles [1]. Many researchers have done a lot of work on that [2-8]. Road standard in our country formulates that the highway minimum plane curve radius is $650 \mathrm{~m}$. Taking the lane line curvature radius of $650 \mathrm{~m}, 40 \mathrm{~m}$ bending lane line in front of the vehicle can be approximate to straight lines. The approximation in most cases can be established from the experiment image, and near-field vision can ensure the vehicle's safety driving. So the linear model is suitable for the standardization highway road detection and near-field vision in the lane line can be obtained by straight lines model fitting.

Lane detection is still a fertile area of machine vision research[9-11]; variations of the Hough transform are still among the most popular and commonly used methods [8]. It transforms the straight line in the image space to corresponding position of a point in the parameter space, and then the position of the point in the parameter space is formed in the peak after cumulative voting. Through the extraction of the peak, the parameters of the line are obtained. In these approaches, the input images are first preprocessed to find edges using a Canny edge detector or steer able filters, followed by a threshold. The classical Hough transform is then used to find straight lines in the binary image.

Classical Hough transformation works well for line finding when the roads are mostly straight; however, it also has shortcomings as the probability density function of the parameters in classical Hough transform is estimated using a discrete two-dimensional histogram [2], Here are two of them: First, in the classical Hough transform, every pixel in the original image space participate in the transformation from image space to parameter space. At the same time, the voting of the parameter point do not always come from the same line in the image space, also may be caused by the chance to arrange of lane marking points which are not in the same straight line. This not only makes the algorithm increased in computational complexity and time-consuming, also lead to the subsequent lower accuracy. Second, for the grid quantization precision in parameter space, classical Hough transform is very difficult to achieve the optimal. If the grid is quantitative too much, that would be result in double counting in a same straight line, thus increasing the computational complexity. If the grid is not quantitative too much, that would be result in a bad space gathering and not getting the right line parameters.

Base on this, the paper models the classical Hough transform parameters by statistical kernel function, and put each pixel's position and gradient orientation in the image as the observation data. The probability density function of the parameters is continuous and contains more useful 
information. This also solves the problem of the grid quantitative accuracy effectively. For processing and modeling on every pixel in the image, computational cost is larger. Using the method of gradient threshold limits the size of initial data set. Only keep large gradient pixels modeling by Gaussian kernel function.

This paper is organized as follows. Section 2 introduces the principle of statistical Hough transform. In section 3, the lane detection method of statistical Hough transforms based on gradient constraint is presented. Results and analyses are given in section 4 , which introduce experimental results on different road situations. Finally, this paper is concluded in section 5 .

\section{The principle of Statistical Hough Transform}

First rewriting the classical Hough transform equation:

$$
\rho=x \cos \theta+y \sin \theta
$$

The variables $x, y, \theta 、 \rho$ are continuous random variables .Some of them may be available in different observation space. In particular, we define the following observation space:

1, $S_{x y}=\left\{\left(x_{i}, y_{i}\right)\right\}_{i=1 \cdots N}$. This is the observation space of variables $(x, y)$ which used in the classical Hough Transform.

2, $S_{\theta x y}=\left\{\left(\theta_{i}, x_{i}, y_{i}\right)\right\}_{i=1 \cdots N}$. This is the observation space of $\theta$. Indeed, when considering images, the gradient orientation can locally be computed and used as an observation $\theta$.

3, $S_{\theta \rho}=\left\{\left(\theta_{i}, \rho_{i}\right)\right\}_{i=1 \cdots N}$.when knowing $\theta_{i}, x_{i}, y_{i}$, the measure $\rho_{i}$ can be computed using (1) and also used as an observation.

In addition to the observations, we attached a prior $p_{i}\left(\sum_{i=1}^{N} p_{i}=1,0 \leq p_{i} \leq 1\right)$ to each observation $i$ 。The statistical framework completely generalizes the classical Hough Transform and also takes advantage of the relation (1) between the random variables. This allows us to propose three different estimates of $p_{\theta \rho}(\theta, \rho)$ for each observation space $\left(S_{x y}, S_{\theta x y}\right.$ and $\left.S_{\theta \rho}\right)$.

Let $G(x, y)$ be the gray image of $f(x, y)$, its first order derivatives are $G_{x}(x, y), G_{y}(x, y)$.The gradient magnitude $\left\|\nabla G_{i}\right\|$ and gradient orientation $\theta_{i}$ for each pixel $i$ at location $\left(x_{i}, y_{i}\right)$ are getting from the following formula:

$$
\begin{aligned}
\left\|\nabla G_{i}\right\| & =\sqrt{G_{x}^{2}\left(x_{i}, y_{i}\right)+G_{y}^{2}\left(x_{i}, y_{i}\right)} \\
\theta_{i} & =\arctan \left(\frac{G_{y}\left(x_{i}, y_{i}\right)}{G_{x}\left(x_{i}, y_{i}\right)}\right)
\end{aligned}
$$

Variance of $\theta_{i}$ is defined as:

$$
\sigma_{\theta_{i}}^{2}=\frac{\sigma^{2}}{\left\|\nabla I_{i}\right\|^{2}}
$$

Where $\sigma^{2}$ is the noise variance, generally defined as 1 .

\subsection{The Probability Density Function Under Different Observation Space}

We propose three estimates of the probability density function $p_{\theta \rho}(\theta, \rho)$ for observation space $S_{\theta \rho}, S_{\theta x y}, S_{x y}$ using kernel modeling.

First, kernel function model is used to give the probability density function $p_{\theta \rho}(\theta, \rho)$ in the observation space $S_{\theta \rho}$.

$$
\hat{p}_{\theta \rho}\left(\theta, \rho \mid S_{\theta \rho}\right)=\sum_{i=1}^{N} \frac{1}{h_{\theta_{i}}} k_{\theta}\left(\frac{\theta-\theta_{i}}{h_{\theta_{i}}}\right) \cdot \frac{1}{h_{\rho_{i}}} k_{\rho}\left(\frac{\rho-\rho_{i}}{h_{\rho_{i}}}\right) p_{i}
$$

Where $h_{\theta_{i}}$ and $h_{\rho_{i}}$ are the variable bandwidths. Their estimations are explained in next section. The kernels $k_{\theta}(\bullet)$ and $k_{\rho}(\bullet)$ have been chosen Gaussians so that (5) gives a continuous and smooth estimate of the density $p_{\theta \rho}(\theta, \rho)$.

For the observation space $S_{\theta x y}$, using the Bayes formula, we can write:

$$
p_{\theta \rho x y}(\theta, \rho, x, y)=p_{\rho \mid \theta x y}(\rho \mid \theta, x, y) p_{\theta x y}(\theta, x, y)
$$

Under the observation space $S_{\theta x y}$, the variable $x, y, \theta$ are known, the variable $\rho$ is deterministic by formula (1). Therefore, we propose to model the conditional probability as follows:

$$
p_{\rho \mid \theta x y}(\rho \mid \theta, x, y)=\delta(\rho-x \cos \theta-y \sin \theta)
$$

Where $\delta(\bullet)$ is the Dirac distribution. As a consequence, only $p_{\theta x y}(\theta, x, y)$ is to estimate using kernels with the observation space $S_{\theta x y}$ :

$$
\hat{p}_{\theta x y}\left(\theta, x, y \mid S_{\theta x y}\right)=\sum_{i=1}^{N} \hat{p}_{\theta x y}\left(\theta, x, y \mid \theta_{i}, x_{i}, y_{i}\right) p_{i}=\sum_{i=1}^{N} \frac{1}{h_{x_{i}}} k_{x}\left(\frac{x-x_{i}}{h_{x_{i}}}\right) \frac{1}{h_{y_{i}}} k_{y}\left(\frac{y-y_{i}}{h_{y_{i}}}\right) \frac{1}{h_{\theta_{i}}} k_{\theta}\left(\frac{\theta-\theta_{i}}{h_{\theta_{i}}}\right) p_{i}
$$

Note that we have assumed the variables $x, y, \theta$ independent given their observation space $\left(\theta_{i}, x_{i}, y_{i}\right)$.The estimate can be computed:

$$
\hat{p}\left(\theta, \rho \mid S_{\theta x y}\right)=\sum_{i=1}^{N} \frac{1}{h_{\theta_{i}}} k_{\theta}\left(\frac{\theta-\theta_{i}}{h_{\theta_{i}}}\right) R_{i}(\theta, \rho) p_{i}
$$


with:

$$
R_{i}(\theta, \rho)=\iint \delta(\rho-x \cos \theta-y \sin \theta) \frac{1}{h_{x_{i}}} k_{x}\left(\frac{x-x_{i}}{h_{x_{i}}}\right) \times \frac{1}{h_{y_{i}}} k_{y}\left(\frac{y-y_{i}}{h_{y_{i}}}\right) d x d y
$$

In the observation space $S_{x y}$ which is the classical Hough transform observation space, the only available thing is the position $(x, y)$. No prior information is available on the variable $\theta$, therefore, its kernel can be replaced in (9) by the uniform distribution as:

$$
\frac{k_{\theta}\left(\theta-\theta_{i}\right)}{h_{\theta_{i}}}=\frac{1}{\pi}
$$

Then, expression (9) becomes:

$$
\hat{p}_{\theta \rho}\left(\theta, \rho \mid S_{x y}\right)=\frac{1}{\pi} \sum_{i} R_{i}(\theta, \rho) p_{i}=\frac{1}{\pi} R(\theta, \rho)
$$

with,

$$
R(\theta, \rho)=\iint \delta(\rho-x \cos \theta-y \sin \theta) \hat{p}_{x y}\left(x, y \mid S_{x y}\right) d x d y
$$

And,

$$
\hat{p}_{x y}\left(x, y \mid S_{x y}\right)=\sum_{i=1}^{N} \frac{1}{h_{x_{i}}} k_{x}\left(\frac{x-x_{i}}{h_{x_{i}}}\right) \frac{1}{h_{y_{i}}} k_{y}\left(\frac{y-y_{i}}{h_{y_{i}}}\right) p_{i}
$$

Equation (10) gives a kernel estimate for the classical Hough transform.

\subsection{Kernels and Bandwidths}

It is usually acknowledged that the choice of the kernel $k(\bullet)$ is very important, we have chosen the kernels $k_{\theta}$ and $k_{\rho}$ as Gaussian and now we discuss the choice of kernels $k_{x}$ and $k_{y}$.Various kernels $k_{x}$ and $k_{y}$ can be used such as:

1. The Dirac kernels are defined as:

$$
\left\{\begin{array}{l}
\frac{k_{x}\left(x-x_{i}\right)}{h_{x_{i}}}=\delta\left(x-x_{i}\right) \\
\frac{k_{y}\left(y-y_{i}\right)}{h_{y_{i}}}=\delta\left(y-y_{i}\right)
\end{array}\right.
$$

In this case, no bandwidths are needed. The corresponding kernel $R(\theta, \rho)$ in the Hough space is then also a Dirac:

$$
R_{i}(\theta, \rho)=\delta\left(\rho-x_{i} \cos \theta-y_{i} \sin \theta\right)
$$

2. The Gaussian kernels are defined as:

$$
\left\{\begin{array}{l}
\frac{k_{x}\left(x-x_{i}\right)}{h_{x_{i}}}=\mathrm{N}\left(x_{i}, h_{x_{i}}^{2}\right) \\
\frac{k_{y}\left(y-y_{i}\right)}{h_{y_{i}}}=\mathrm{N}\left(y_{i}, h_{y_{i}}^{2}\right)
\end{array}\right.
$$

The corresponding kernel $R(\theta, \rho)$ in the Hough space is:

$$
G_{i}(\rho, \theta)=\frac{1}{\sqrt{2 \pi\left(\sigma_{x_{i}}^{2} \cos ^{2} \theta+\sigma_{y_{i}}^{2} \sin \theta\right)}} \exp \left(\frac{-\left(\rho-x_{i} \cos \theta-y_{i} \sin \theta\right)^{2}}{2\left(\sigma_{x_{i}}^{2} \cos ^{2} \theta+\sigma_{y_{i}}^{2} \sin ^{2} \theta\right)}\right)
$$

In this paper, we have chosen the kernels as Gaussian. The Dirac kernel is then just a special case of the Gaussian kernel when the bandwidth goes toward 0 .

For the variables $\theta$ and $\rho$, we naturally set the bandwidths $\quad h_{q_{i}}=s_{q_{i}} \quad, \quad h_{r_{i}}=s_{r_{i}} \quad$.The bandwidths $\left(h_{x_{i}}, h_{y_{i}}\right)$ are estimated in a similar fashion as the variable bandwidth for $\theta$. $\left(h_{x_{i}}, h_{y_{i}}\right)$ reflect the uncertainty attached to the observation space $\left(x_{i}, y_{i}\right)$ of the variables $(x, y)$. Because of the digitalization process, the observation spaces of the variables $(x, y)$ have a precision \pm 1 . Then, we set

$$
h_{x_{i}}=h_{y_{i}}=1
$$

\section{Lane Detection Method of Statistical Hough Transform Based on Gradient Constraint}

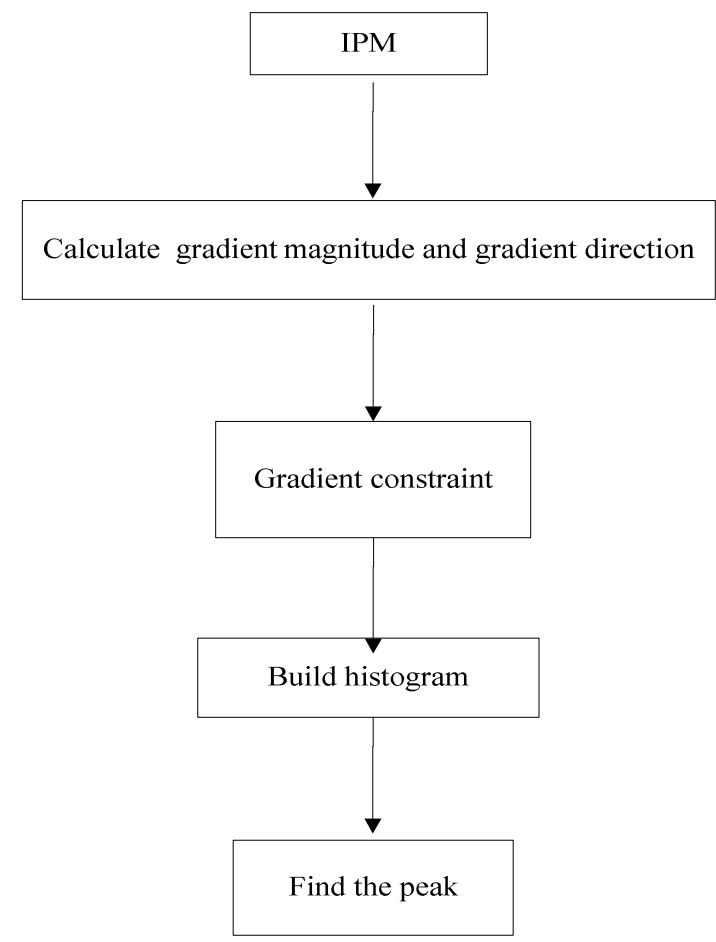

Figure 1. The flow chart of lane detection algorithm. 
Based on the statistical Hough transform method described in the previous section, we implement a robust lane detection method based on gradient constraint. The proposed method starts from an inverse perspective mapping algorithm, then each pixel gradient magnitude and gradient orientation are calculated. The gradient constraint method is performed as a selection step, when the pixel gradient magnitude is greater than the threshold; this pixel is selected to model by Gaussian kernel function. Then build a 3D histogram based on the probability density function. The peaks in the histogram are the desired lane parameters. The flow chart of lane detection algorithm is shown in Fig.1.

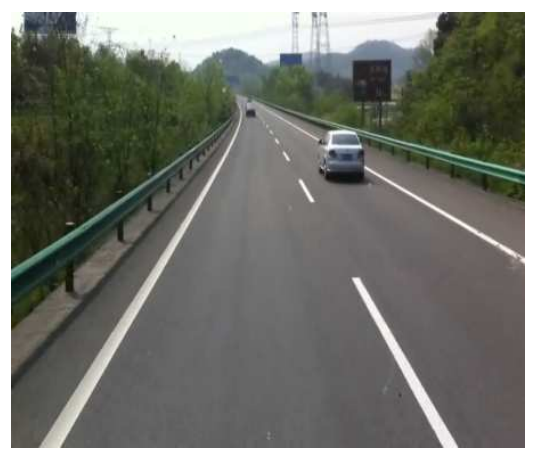

(a)

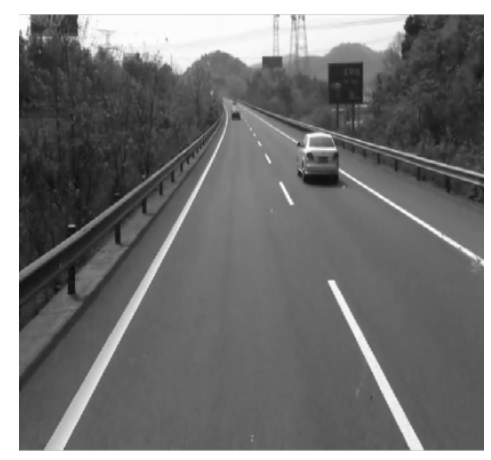

(b)

\subsection{IPM}

Based on the flat ground hypothesis with known extrinsic and intrinsic parameters of the camera, IPM can remove the perspective effect. It remaps pixels from the original image to the other image that has a different coordinate system. This remapping procedure can be done by a fast lookup table with distortion compensation. As the resolution for near and further object is different in the original image, an interpolation process is needed in the IPM algorithm [3][4]. The resulting image is shown in Fig. 2.We can see from Fig 2 that IPM creates a somewhat cleaned up and less complex image where distracters (sky, objects, etc.,) are downplayed.

Figure 2. (a) Original image. (b) Gray image. (c)IPM image.

\subsection{Gradient Constraint}

The probability density function of lane parameters is estimated by multiple kernel density, and the uncertain variables are modeled by Gaussian kernels. Statistical Hough transform can directly work on the gray image and it is more robust than the classical Hough transform against noise. But computing the Statistical Hough Transform on an image is more computationally expensive than the classical Hough transform because all pixels of the image are used. The gradient constraint method used to decrease the number of the pixel, in addition, would not cause histogram sparse.

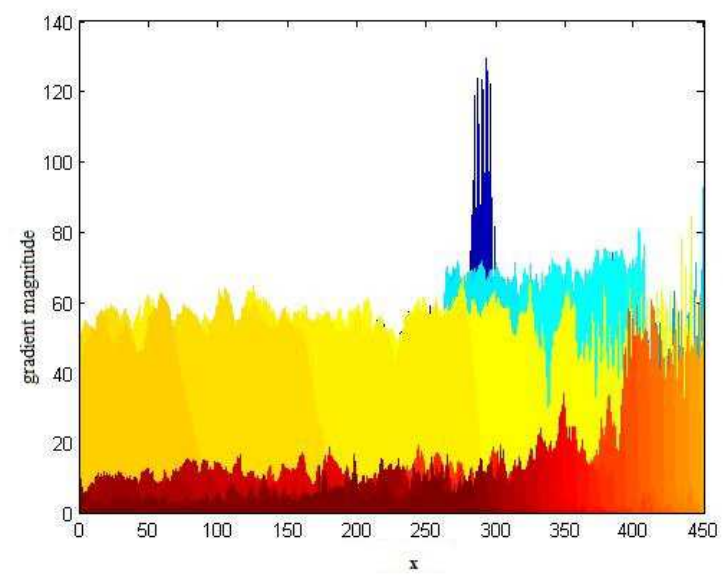

Figure 3. Gradient distribution of Figure 2-c.

Here, we analysis gradient magnitude of the image and get the distribution figure of it, as shown in fig.3. We can get the minimum and maximum gradient magnitude is 0 and 126,respectively. Most of the pixels' gradient magnitudes in the image are relatively small. The number of pixel with gradient magnitude varies from 0 to 20 is the most. These pixels not only increase the complexity of modeling, but also have little chance on the lane line. So, we take those pixels as the basis pixels and get rid of the pixel whose gradient magnitude is zero to calculate the gradient magnitude threshold $T$. Pixels whose gradient magnitude is greater than the threshold is to participate in statistics Hough transform. The threshold is obtained as:

$$
T=G_{\min }+\frac{\left|G_{\max }-G_{\min }\right|}{10}
$$

Where $G_{\min }$ is the minimum gradient magnitude, $G_{\max }$ is the maximum gradient magnitude. Here, we define $G_{\min }=20, G_{\min }$ value can decide by the actual gradient magnitude range.

Introducing the gradient constraint method to statistical Hough transform on the one hand keeps the on lane line pixels modeled by Gaussian as far as possible, on the other hand reduces the amount of calculation of building histogram.

\subsection{Build Histogram}

This section we applied the kernels function to lane detection. Distribution of lane parameters is determined by 
the position of each pixel in the image given by $\left(x_{i}, y_{i}\right)$ and the orientation of the pixel $\theta_{i}$, giving the observation space $Q_{x y \theta}$, the lane parameters probability density function can be represented as $p\left(\rho, \theta \mid Q_{x y \theta}\right)$.It can be written according to Bayes rule [3]:

$$
p\left(\rho, \theta, x, y \mid S_{x y \theta}\right)=p\left(\rho \mid x, y, \theta, S_{x y \theta}\right) p\left(x, y, \theta \mid S_{x y \theta}\right)
$$

In (19), the first probability $p\left(r \mid x, y, q, S_{x y q}\right)$ is determined by (1), and the second probability $p\left(x, y, q \mid S_{x y q}\right)$ can be modeled by a Gaussian kernel function, thus (19) becomes:

$$
p\left(\rho, \theta, x, y \mid S_{x y \theta}\right)=\delta(\rho-x \cos \theta-y \sin \theta) \frac{1}{N} \sum_{i} K_{x} K_{y} K_{\theta}
$$

Where $\delta(\bullet)$ is the Dirac function, $K_{x} \sim \mu\left(x_{i}, \sigma_{x_{i}}^{2}\right), K_{y} \sim \mu\left(y_{i}, \sigma_{y_{i}}^{2}\right), K_{\theta} \sim \mu\left(\theta_{i}, \sigma_{\theta_{i}}^{2}\right) \quad$ are Gaussian kernels. The distribution $p\left(r, q \mid S_{x y q}\right)$ can be obtained by integrating (20) over (x, y):

$$
p\left(\rho, \theta \mid S_{x y \theta}\right)=\frac{1}{N} \sum_{i} G_{i}(\rho, \theta)
$$

Where

$$
G_{i}(\rho, \theta)=\frac{1}{\sqrt{2 \pi\left(\sigma_{x_{i}}^{2} \cos ^{2} \theta+\sigma_{y_{i}}^{2} \sin ^{2} \theta\right)}} \exp \left(\frac{-\left(\rho-x_{i} \cos \theta-y_{i} \sin \theta\right)^{2}}{2\left(\sigma_{x_{i}}^{2} \cos ^{2} \theta+\sigma_{y_{i}}^{2} \sin ^{2} \theta\right)}\right)
$$

Let

$$
\theta \in\left[-\frac{\pi}{2}, \frac{\pi}{2}\right] \quad, \quad \rho \in\left[-\rho_{j}, \rho_{j}\right] \quad, \quad \text { where }
$$

$\rho_{j}=\frac{w}{2} \cos \left(a \tan \left(\frac{h}{w}\right)\right)+\frac{h}{2} \sin \left(a \tan \left(\frac{h}{w}\right)\right)$, And $\mathrm{w}$ and $\mathrm{h}$ are the width and height of the IPM image. Then compute the probability for selected pixels according to (21). The variance of $x, y, \theta$ are defined as $\sigma_{x_{i}}^{2}=1, \sigma_{y_{i}}^{2}=1, \sigma_{\theta_{i}}^{2}=\frac{1}{\left\|\nabla G_{i}\right\|^{2}}$ 。

Fig.4 shows the histogram of fig.2-c.

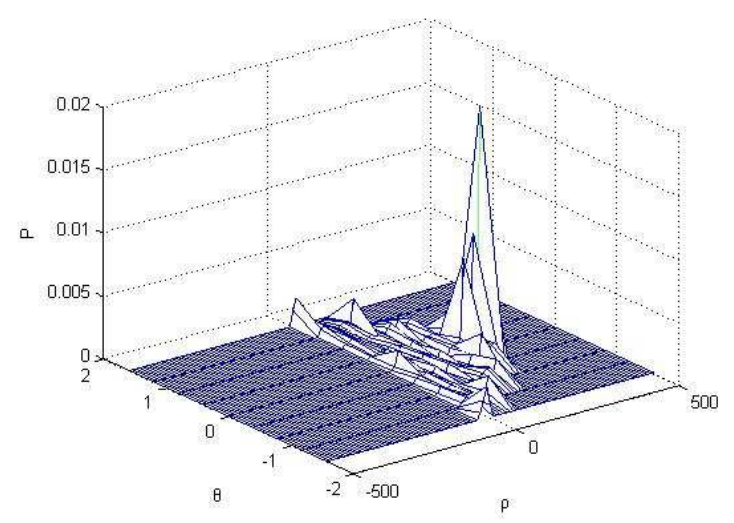

Figure 4. Histogram of statistics Hough transforms.

\section{Results and Analyses}

Test images on our experiments are captured using on-board system. To demonstrate the performance of the algorithm, we apply it on various situations on the highway under the environment of laboratory. This paper selected 3000 frames of images under various scenarios for testing, a total of 2780 frames correctly detect the lane line, and the correct detection probability is up to $92.7 \%$.

Figure 5 contains two groups' lane detection results, each group consists two parts, the upper part is the original image, and the lower part is the test results. Group (a) is the detection result of no obstacle in the vision field; Group (b) is the detection result of cars or other obstacles occlude in the lane. As can be seen from the test results, under different highway situations, the proposed method detects the lane location in the real range, in case of a small amount of vehicles or obstacle; the robust algorithm can still detect the lane line correctly.
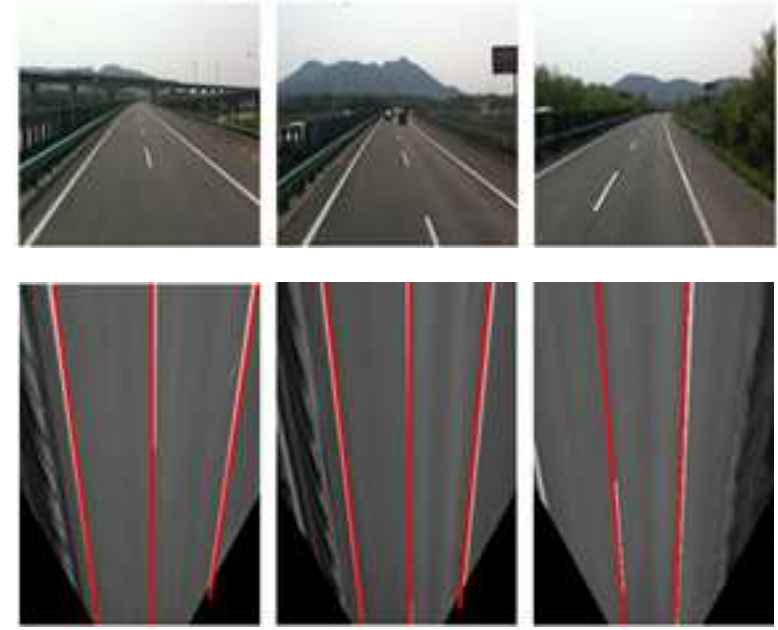

(a)
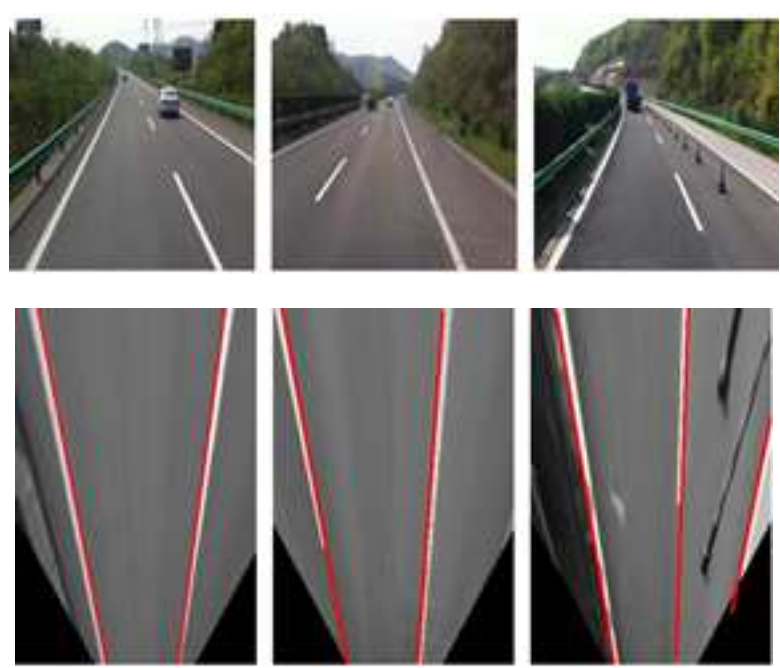

(b)

Figure 5. Lane detection results.

Using the gradient constraint method limits the size of 
initial data set. Only keep large gradient magnitude pixels modeling by Gaussian kernel function to reducing the amount of calculation. Tab. 1 shows the processing speed and the total number of pixels participates in the histogram before and after gradient constraint for Fig.2-c. Before gradient constraint, the processing speed is $1.1 \mathrm{~s}$ per frame, after gradient constraint, the processing speed is $0.64 \mathrm{~s}$ per frame. The detection results in Fig 5 reveal the gradient constraint method does not affect the validity of the results.

Table 1. Total number of pixels and processing time contrast before and after gradient constraint.

\begin{tabular}{lll}
\hline & total number & processing speed \\
\hline Before gradient constraint & 127600 & 1.10 second/per frame \\
After gradient constraint & 53208 & 0.64 second/per frame \\
\hline
\end{tabular}

\section{Conclude}

A lane detection method of Statistical Hough transform based on gradient constraint is proposed on analyzes the advantages and disadvantages of classical Hough transform. Statistical Hough transform uses the Gaussian kernel function to model each pixel in the image solving the problem of the classical Hough transform grid quantization accuracy. Using the method of gradient constraint limits the size of initial data set for reducing the amount of calculation and improving the follow-up detecting accuracy. Eventually lane parameters of continuous probability density function are given. The results of the experimentation show that under highway circumstance the provided method can rapidly and robustly detect the lane.

\section{References}

[1] Aharon Bar Hillel, Ronen Lerner, Danlevi, Guy Raz. "Recent progress in road and lane detection: a survey", Machine Vision and Application, vol. 10, no. 4, pp.727-745, 2014.

[2] Rozenn Dahyot, "Statistical Hough transform", IEEE Transactions on pattern analysis and machine intelligence, vol. 31, no.8, pp.1502-1509, 2009.

[3] G.Liu, F.Wörgötter, I.Markelic', "combining statistical Hough transform and particle filter for rubust lane detection and tracking", In Proceeding(s) of IEEE Intelligent Vehicles Symposinm University of California,pp.993-997,2010

[4] G.Liu, F.Wörgötter, I.arkeli, "Stochastic lane shape estimation using local image descriptors", IEEE Transactions on intelligent transportation systems, IEEE, vol. 14, no. 1, pp.13-21, 2013.

[5] H.Yoo, U.Yang, K.Sohn, "Gradient-enhancing conversion for illumination-robust lane detection", IEEE Transactions on intelligent transportation systems, IEEE, vol. 14, no.3, pp.1083-1094, 2013.

[6] R.Gopalan,T.Hong,M.Shneier. "A learning approach towards detection and tracking of lane markings", IEEE Transactions on intelligent transportation systems, IEEE, vol. 13, no.3, pp.1088-1098, 2012.

[7] Z.Kim. "Robust lane detection and tracking in challenging scenarios", IEEE Transactions on intelligent transportation systems, IEEE, vol. 9, no.1, pp.16-26, 2008.

[8] Amol Borkar, Monson Hayes,Mark T.Smith. "A novel lane detection system with efficient ground truth generation", IEEE Transactions on intelligent transportation systems, IEEE, vol.13, no.1, pp.365-374, 2012.

[9] J.C.McCall, M.M.Triedi, "Video based lane estimation and tracking for driver assistance:Survey,system and evaluation". IEEE Transactions on intelligent transportation systems, IEEE, vol.7, no.1, pp.20-37, 2006.

[10] Guangtao Cui, Junzheng Wang, Jing Li, "Robust multilane detection and tracking in urban scenarios based on LIDAR and mono-vision ", IET Image Processing, vol.8, no.5, pp.269-279, 2014.

[11] Y Wang, N.Dahnoun, A.Achim. "A novel system for robust lane detection and tracking".Signal Processing, vol.92, no.2, pp.319-334, 2012. 